\title{
On the nuclearity of certain Cuntz-Pimsner algebras
}

\author{
FERNANDO LLEDÓ \\ Institute for Pure and Applied Mathematics, \\ RWTH-Aachen, Templergraben 55, \\ D-52062 Aachen, Germany. \\ lledo@iram.rwth-aachen.de
}

\author{
EZIo VASSELLi \\ Dipartimento di Matematica, \\ University of Rome "La Sapienza" \\ P.le Aldo Moro 2, I-00185 Roma, Italy \\ vasselli@mat.uniroma2.it
}

September 22, 2018

\begin{abstract}
In the present paper, we give a short proof of the nuclearity property of a class of CuntzPimsner algebras associated with a Hilbert $\mathcal{A}$-bimodule $\mathcal{M}$, where $\mathcal{A}$ is a separable and nuclear $C^{*}$-algebra. We assume that the left $\mathcal{A}$-action on the bimodule $\mathcal{M}$ is given in terms of compact module operators and that $\mathcal{M}$ is direct summand of the standard Hilbert module over $\mathcal{A}$.
\end{abstract}

\section{Introduction}

M.V. Pimsner introduced in his seminal paper [15] a new family of $C^{*}$-algebras $\mathcal{O}_{\mathcal{M}}$ that are naturally generated by a Hilbert bimodule $\mathcal{M}$ over a $C^{*}$-algebra $\mathcal{A}$. These algebras generalise Cuntz-Krieger algebras as well as crossed-products by the group $\mathbb{Z}$. In Pimsner's construction $\mathcal{O}_{\mathcal{M}}$ is given as a quotient of a Toeplitz like algebra acting on a concrete Fock space associated to $\mathcal{M}$. An alternative abstract approach to Cuntz-Pimsner algebras in terms of $C^{*}$-categories is given in [8, 12, 16] (for the notion of $\mathrm{C}^{*}$-category see [10]).

In the present note, we give a short proof of nuclearity property of the Cuntz-Pimsner algebra (cf. Theorem 2.7) associated with a full Hilbert bimodule $\mathcal{M}$ with faithful left action, satisfying the following additional properties:

(i) The coefficient $C^{*}$-algebra $\mathcal{A}$ is nuclear and separable.

(ii) The left $\mathcal{A}$-action is given in terms of compact module operators and is non-degenerate.

(iii) $\mathcal{M}$ is a direct summand in the standard Hilbert module $\mathfrak{H}_{\mathcal{A}}$ over $\mathcal{A}$.

Nuclearity of Cuntz-Pimsner algebras has been discussed recently in the concrete Toeplitz algebra setting (cf. [13, 11]). The paper by Kumjian is in a certain sense complementary to ours. In fact, he considers left $\mathcal{A}$-actions that have a trivial intersection with the compact module operators. Our proof uses the alternative approach in [8]. In particular, we analyse the structure of some spectral subspaces $\mathcal{O}_{\mathcal{M}}^{k}, k \in \mathbb{N}_{0}$, that are associated to a natural circle action. An important step in the proof is to recognize the structure of $\mathcal{O}_{\mathcal{M}}^{1}$ as an imprimitivity $\mathcal{O}_{\mathcal{M}^{-}}^{0}$ bimodule. In this way we can apply a result by Brown, Green and Rieffel to the corresponding stabilizations and consider, roughly speaking, the Cuntz-Pimsner algebra as a crossed-product of the zero-grade spectral subspace $\mathcal{O}_{\mathcal{M}}^{0}$, which is shown to be a nuclear $C^{*}$-algebra. 


\section{Basic definitions and the main theorem}

Let $\mathcal{A}$ be a $C^{*}$-algebra and $\mathcal{M}$ a Hilbert $\mathcal{A}$-module. We denote by $\mathcal{L}(\mathcal{M})$ the $C^{*}$-algebra of adjointable, right $\mathcal{A}$-module operators on $\mathcal{M}$ and by $\mathcal{K}(\mathcal{M}) \subseteq \mathcal{L}(\mathcal{M})$ the (closed) ideal of compact operators generated by the maps

$$
\theta_{\psi, \psi^{\prime}} \in \mathcal{L}(\mathcal{M}), \quad \psi, \psi^{\prime} \in \mathcal{M}, \quad \text { with } \quad \theta_{\psi, \psi^{\prime}}(\varphi):=\psi\left\langle\psi^{\prime}, \varphi\right\rangle, \varphi \in \mathcal{M}
$$

where $\langle\cdot, \cdot\rangle$ is the $\mathcal{A}$-valued scalar product defined on $\mathcal{M}$.

We denote by $\mathfrak{H}_{\mathcal{A}}$ the standard (countably generated) Hilbert $\mathcal{A}$-module of sequences $\left(A_{n}\right)_{n}$ such that $\sum_{n} A_{n}^{*} A_{n}$ converges in $\mathcal{A}$ (cf. [4, Example 13.1.2 (c)]). It is well-known that the $C^{*}$ algebra $\mathcal{K}\left(\mathfrak{H}_{\mathcal{A}}\right)$ of compact, right $\mathcal{A}$-module operators on $\mathfrak{H}_{\mathcal{A}}$ is isomorphic to $\mathcal{K} \otimes \mathcal{A}$, where $\mathcal{K}$ is the $C^{*}$-algebra of compact operators over a separable Hilbert space. The multiplier algebra of $\mathcal{K}\left(\mathfrak{H}_{\mathcal{A}}\right)$ is isomorphic to $\mathcal{L}\left(\mathfrak{H}_{\mathcal{A}}\right)$. We will regard the standard module $\mathfrak{H}_{\mathcal{A}}$ as a Hilbert $\mathcal{A}$-bimodule with the obvious left $\mathcal{A}$-module action.

In this paper, we will consider Hilbert $\mathcal{A}$-modules $\mathcal{M}$ which are direct summands of $\mathfrak{H}_{\mathcal{A}}$. This implies that $\mathcal{M}$ is finitely or countably generated. Conversely by Kasparov stabilization every countably generated Hilbert $\mathcal{A}$-module is a direct summand of $\mathfrak{H}_{\mathcal{A}}$. Moreover, if $\mathcal{A}$ is unital, then also every algebraically finitely generated Hilbert $\mathcal{A}$-module is a direct summand of $\mathfrak{H}_{\mathcal{A}}$. The left $\mathcal{A}$-action on $\mathcal{M}$ is given by a ${ }^{*}$-homomorphism $\alpha: \mathcal{A} \rightarrow \mathcal{L}(\mathcal{M})$. In the present paper, we will assume that $\alpha$ is faithful (in the sequel, we will identify elements of $\mathcal{A}$ with their image in $\mathcal{L}(\mathcal{M})$ ) and with image contained in $\mathcal{K}(\mathcal{M})$. We will also assume that $\alpha$ is non-degenerate in the sense that

$$
\mathcal{A} \cdot \mathcal{K}(\mathcal{M}):=\text { closed } \operatorname{span}\{A T \mid A \in \mathcal{A}, T \in \mathcal{K}(\mathcal{M})\}=\mathcal{K}(\mathcal{M}) .
$$

Note that when $\mathcal{M}$ is algebraically finitely generated, then $\mathcal{L}(\mathcal{M})=\mathcal{K}(\mathcal{M})$, thus every left $\mathcal{A}$-action is given by compact module operators.

We denote by $\mathcal{O}_{\mathcal{M}}$ the Cuntz-Pimsner algebra associated with the Hilbert bimodule $\mathcal{M}$ (cf. [15]). Recall that $\mathcal{O}_{\mathcal{M}}$ is generated as a $C^{*}$-algebra by $\mathcal{M}$ and $\mathcal{A}$ satisfying the relations

$$
\begin{aligned}
\psi^{*} \psi^{\prime} & =\left\langle\psi, \psi^{\prime}\right\rangle, \quad \psi, \psi^{\prime} \in \mathcal{M} \\
A \psi & :=\alpha(A) \psi, \quad A \in \mathcal{A} .
\end{aligned}
$$

Note that (3) implies $\psi^{\prime} \psi^{*} \varphi=\theta_{\psi^{\prime}, \psi}(\varphi), \psi^{\prime}, \psi^{*}, \varphi \in \mathcal{M}$. Therefore one has the natural identification

$$
\theta_{\psi^{\prime}, \psi}=\psi^{\prime} \psi^{*} \text {. }
$$

Moreover, if $\mathcal{M}^{r}:=\mathcal{M} \otimes_{\mathcal{A}} \cdots \otimes_{\mathcal{A}} \mathcal{M}, r \in \mathbb{N}$, is the $r$-fold tensor product with coefficients in $\mathcal{A}$, then there is an identification

$$
\mathcal{M}^{r} \simeq \text { closed } \operatorname{span}\left\{\psi_{1} \cdot \ldots \cdot \psi_{r} \in \mathcal{O}_{\mathcal{M}} \mid \psi_{k} \in \mathcal{M}, k=1, \ldots, r\right\}
$$

There is a natural action of the circle $\mathbb{T}:=\{z \in \mathbb{C}|| z \mid=1\}$ on the Cuntz-Pimsner algebra given by

$$
\delta: \mathbb{T} \rightarrow \operatorname{Aut} \mathcal{O}_{\mathcal{M}}, \quad \delta_{z}(\psi):=z \psi \quad, \quad z \in \mathbb{T}, \psi \in \mathcal{M}
$$

We denote by

$$
\mathcal{O}_{\mathcal{M}}^{k}:=\left\{T \in \mathcal{O}_{\mathcal{M}} \mid \delta_{z}(T)=z^{k} T, z \in \mathbb{T}\right\}, k \in \mathbb{Z},
$$

the spectral subspaces associated to the circle action. In particular, $\mathcal{O}_{\mathcal{M}}^{0}$ is the closed span of elements of the form $\psi^{\prime} \psi^{*}, \psi, \psi^{\prime} \in \mathcal{M}^{r}, r \in \mathbb{N}$. From Eq. (2) we have that

$$
\mathcal{A} \cdot \mathcal{M}:=\text { closed } \operatorname{span}\{A \psi \mid A \in \mathcal{A}, \psi \in \mathcal{M}\}=\mathcal{M} .
$$


(Use the fact that every $\psi \in \mathcal{M}$ is of the form $\psi=T \psi^{\prime}$ for some $T \in \mathcal{K}(\mathcal{M}), \psi^{\prime} \in \mathcal{M}$, cf. $[\underline{5}$, Lemma 1.3]). Therefore $\mathcal{A} \cdot \mathcal{M}^{r}=\mathcal{M}^{r}, r \in \mathbb{N}$, and

$$
\mathcal{A} \cdot \mathcal{O}_{\mathcal{M}}^{0}=\text { closed } \operatorname{span}\left\{A T \mid A \in \mathcal{A}, T \in \mathcal{O}_{\mathcal{M}}^{0}\right\}=\mathcal{O}_{\mathcal{M}}^{0}
$$

In order to discuss universality properties of the Cuntz-Pimsner algebra we give the following definitions (cf. [ㅇ, §2])

Definition 2.1 Let $\mathcal{A} \subset \mathcal{B}$ be an inclusion of $C^{*}$-algebras. $A$ Hilbert $\mathcal{A}$-bimodule in $\mathcal{B}$ is a closed vector space $\mathcal{M} \subset \mathcal{B}$ satisfying

(i) $A \psi \in \mathcal{M}, \psi A \in \mathcal{M}$ and $\psi^{*} \psi^{\prime} \in \mathcal{A}$ for every $A \in \mathcal{A}, \psi, \psi^{\prime} \in \mathcal{M}$.

(ii) For any $A \in \mathcal{A}$ with $A \psi=0, \psi \in \mathcal{M}$, one has $A=0$.

We say that $\mathcal{M}$ is full if for every $A \in \mathcal{A}$ there are $\psi, \psi^{\prime} \in \mathcal{M}$ such that $A=\psi^{*} \psi^{\prime}$.

Now, there is a natural identification

$$
\mathcal{M M}^{*}:=\text { closed } \operatorname{span}\left\{\psi^{\prime} \cdot \psi^{*} \mid \psi, \psi^{\prime} \in \mathcal{M} \subset \mathcal{B}\right\} \simeq \mathcal{K}(\mathcal{M}),
$$

hence $\mathcal{M M}^{*}$ may be regarded as a $C^{*}$-subalgebra of $\mathcal{B}$. We say that $\mathcal{M}$ has support $\mathbb{1}$ if there exists a sequence $\left\{\psi_{n}\right\} \subset \mathcal{M}$ such that $\left\{U_{N}:=\sum_{n=1}^{N} \psi_{n} \psi_{n}^{*}\right\}_{N}$ is an approximate unit for $\mathcal{B}$ (recall that by assumption the $C^{*}$-algebras are separable.)

Remark 2.2 Note that if $\mathcal{M} \subset \mathcal{B}$ has support $\mathbb{1}$ and if there are elements $A \in \mathcal{A}, T \in \mathcal{M M}^{*}$ satisfying $A \psi=T \psi$ for every $\psi \in \mathcal{M}$, then

$$
T=A
$$

In fact, since $T \psi_{n}=A \psi_{n}$ for every $n \in \mathbb{N}$, we have $T U_{N}=A U_{N}$ for every $N \in \mathbb{N}$ and therefore

$$
T=\lim _{N} T U_{N}=\lim _{N} A U_{N}=A .
$$

The following result is just a translation of [15, Theorem 3.12] in terms of Hilbert bimodules in $C^{*}$-algebras. We note explicitely that Eq. (10) is equivalent to condition (4) in the above-cited theorem.

Proposition 2.3 Let $\mathcal{A} \subset \mathcal{B}$ be an inclusion of unital $C^{*}$-algebras, $\mathcal{M} \subset \mathcal{B}$ a full Hilbert $\mathcal{A}$ bimodule in $\mathcal{B}$ with support $\mathbb{1}$. Then there is a canonical morphism $\mathcal{O}_{\mathcal{M}} \rightarrow \mathcal{B}$.

Remark 2.4 Examples of the above universality property can be found in the Cuntz-Pimsner algebra itself. In fact, if $\mathcal{M}$ is algebraically finitely generated and $\mathcal{A}$ is unital, then it follows from the definition of the Cuntz-Pimsner algebra that $\sum_{n} \psi_{n} \psi_{n}^{*}=\mathbb{1}$ for every finite set $\left\{\psi_{n}\right\}$ of (normalized) generators of $\mathcal{M}$. If $\mathcal{M}$ is countably generated as a right $\mathcal{A}$-module, then there are elements $\left\{\psi_{n}\right\}_{n=1}^{\infty} \subset \mathcal{M}$ such that $U_{N}:=\sum_{n=1}^{N} \psi_{n} \psi_{n}^{*}$ is an approximate unit for $\mathcal{K}(\mathcal{M})$, hence also for $\mathcal{A}$ which may be regarded as a $C^{*}$-subalgebra of $\mathcal{K}(\mathcal{M})$ (see for example [6] or p. 266 in [8]). Finally, since $\mathcal{O}_{\mathcal{M}}$ is generated as a $C^{*}$-algebra by $\mathcal{M}$ and $\mathcal{A}$, we conclude that $\left\{U_{N}\right\}_{N}$ is an approximate unit for $\mathcal{O}_{\mathcal{M}}$, so that $\mathcal{M}$ has support $\mathbb{1}$ in $\mathcal{O}_{\mathcal{M}}$.

Remark 2.5 The $C^{*}$-algebra $\mathcal{K}$ of compact operators over a separable Hilbert space is clearly a Hilbert $\mathcal{K}$-bimodule with left and right actions defined by multiplication and scalar product $\left\langle V, V^{\prime}\right\rangle:=V^{*} V^{\prime}, V, V^{\prime} \in \mathcal{K}$. Let $\mathcal{M}$ be a Hilbert $\mathcal{A}$-bimodule. We consider the external tensor product of Hilbert bimodules $\mathcal{M} \widehat{\otimes} \mathcal{K}$, which has a natural structure of Hilbert $(\mathcal{A} \otimes \mathcal{K})$-bimodule, and denote by $\mathcal{O}_{\mathcal{M} \widehat{\otimes} \mathcal{K}}$ the associated Cuntz-Pimsner algebra. Then, there is a natural identification $\mathcal{O}_{\mathcal{M} \widehat{\otimes} \mathcal{K}} \simeq \mathcal{O}_{\mathcal{M}} \otimes \mathcal{K}$ defined by the map $\psi \widehat{\otimes} V \mapsto \psi \otimes V, \psi \in \mathcal{M}, V \in \mathcal{K}$. 
Proposition 2.6 Let $\mathcal{M}$ be a Hilbert $\mathcal{A}$-bimodule satisfying properties (i)-(iii) of the introduction and let $\mathcal{O}_{\mathcal{M}}$ be the corresponding Cuntz-Pimsner algebra. Then the zero grade $C^{*}$-algebra $\mathcal{O}_{\mathcal{M}}^{0}$ (cf. (8)) is nuclear.

Proof: First we prove that every $\mathcal{K}\left(\mathcal{M}^{r}\right), r \in \mathbb{N}$, is a nuclear $C^{*}$-algebra. Since $\mathcal{M}$ is countably generated (or algebraically finitely generated if $\mathcal{A}$ is unital), we conclude that $\mathcal{M}^{r}$ is countably generated (or algebraically finitely generated if $\mathcal{A}$ is unital). By Kasparov stabilization, we obtain that $\mathcal{M}^{r}$ is a direct summand of $\mathfrak{H}_{\mathcal{A}}$. This implies that $\mathcal{K}\left(\mathcal{M}^{r}\right)$ is a corner of the nuclear $C^{*}$ algebra $\mathcal{K}\left(\mathfrak{H}_{\mathcal{A}}\right) \simeq \mathcal{A} \otimes \mathcal{K}$ and, therefore, $\mathcal{K}\left(\mathcal{M}^{r}\right)$ is nuclear. Now, for every $r \in \mathbb{N}$ there is an embedding

$$
i_{r}: \mathcal{K}\left(\mathcal{M}^{r}\right) \rightarrow \mathcal{L}\left(\mathcal{M}^{r+1}\right), \quad i_{r}(T) \psi \varphi:=(T \psi) \varphi,
$$

$\psi \in \mathcal{M}^{r}, \varphi \in \mathcal{M}$ (in the usual tensor notation the embedding is given simply by $i_{r}(T):=T \otimes \mathbb{1}$, where $\mathbb{1}$ is the identity of $\mathcal{L}(\mathcal{M}))$. Next we show that if the image of the left $\mathcal{A}$-action is contained in $\mathcal{K}(\mathcal{M})$, then $i_{r}(T) \in \mathcal{K}\left(\mathcal{M}^{r+1}\right)$. First put $T:=\psi_{1} \psi_{2}^{*}, \psi_{1}, \psi_{2} \in \mathcal{M}^{r}$ so that, $i_{r}(T) \psi \varphi=\psi_{1} \psi_{2}^{*} \psi \varphi$. By [5, Lemma 1.3], there is a decomposition $\psi_{1}=\psi_{0} A_{0}$ for some $A_{0} \in \mathcal{A}$, $\psi_{0} \in \mathcal{M}^{r}$. Moreover, since the left $\mathcal{A}$-module action is given by compact operators, there exist $\varphi_{0}, \varphi_{1} \in \mathcal{M}$ with $A_{0}=\varphi_{0} \varphi_{1}^{*}$, hence $i_{r}(T)=\psi_{0} \varphi_{0} \varphi_{1}^{*} \psi_{2}^{*}$ is an element of $\mathcal{K}\left(\mathcal{M}^{r+1}\right)$. Therefore the zero grade algebra $\mathcal{O}_{\mathcal{M}}^{0}$ is an inductive limit

$$
\mathcal{O}_{\mathcal{M}}^{0}=\lim _{\longrightarrow}\left(\mathcal{K}\left(\mathcal{M}^{r}\right), i_{r}\right)
$$

Since every $\mathcal{K}\left(\mathcal{M}^{r}\right)$ is nuclear, we conclude that $\mathcal{O}_{\mathcal{M}}^{0}$ is nuclear.

We can now prove our main theorem.

Theorem 2.7 Let $\mathcal{A}$ be a nuclear and separable $C^{*}$-algebra. Assume that $\mathcal{M}$ is a full and nondegenerate Hilbert $\mathcal{A}$-bimodule with faithful left $\mathcal{A}$-action and satisfying:

(i) $\mathcal{M}$ is a direct summand (as a right Hilbert $\mathcal{A}$-module) of the standard Hilbert module $\mathfrak{H}_{\mathcal{A}}$ over $\mathcal{A}$.

(ii) The left $\mathcal{A}$-action on $\mathcal{M}$ is given in terms of compact module operators.

Then the Cuntz-Pimsner algebra $\mathcal{O}_{\mathcal{M}}$ is nuclear.

Proof: Consider the spectral subspace $\mathcal{O}_{\mathcal{M}}^{1} \subset \mathcal{O}_{\mathcal{M}}$ which was introduced in (8) and note that it has a natural structure as a $\mathcal{O}_{\mathcal{M}}^{0}$-bimodule. In fact, take left and right multiplication by elements of $\mathcal{O}_{\mathcal{M}}^{0} \subset \mathcal{O}_{\mathcal{M}}$ and define the $\mathcal{O}_{\mathcal{M}}^{0}$-valued scalar product by

$$
\left\langle T, T^{\prime}\right\rangle:=T^{*} T^{\prime}, \quad T, T^{\prime} \in \mathcal{O}_{\mathcal{M}}^{1} .
$$

We show next that $\mathcal{O}_{\mathcal{M}}^{1}$ is a full Hilbert $\mathcal{O}_{\mathcal{M}}^{0}$-module: by Eq. (9) we have that $\mathcal{A} \cdot \mathcal{O}_{\mathcal{M}}^{0}=\mathcal{O}_{\mathcal{M}}^{0}$, so that any $T \in \mathcal{O}_{\mathcal{M}}^{0}$ can be written as $T=A T^{\prime}$ for some $A \in \mathcal{A}$ and $T^{\prime} \in \mathcal{O}_{\mathcal{M}}^{0}$. Since $\mathcal{M}$ is full, there are $\psi, \psi^{\prime} \in \mathcal{M}$ such that $A=\psi^{*} \psi^{\prime}$ and therefore

$$
T=A T^{\prime}=\psi^{*} \psi^{\prime} T^{\prime}=\left\langle\psi, \psi^{\prime} T^{\prime}\right\rangle \in\left\langle\mathcal{O}_{\mathcal{M}}^{1}, \mathcal{O}_{\mathcal{M}}^{1}\right\rangle
$$

Therefore $\mathcal{O}_{\mathcal{M}}^{1}$ is full as a Hilbert $\mathcal{O}_{\mathcal{M}}^{0}$-module.

Denote by

$$
\mathfrak{O}:=\mathcal{O}_{\mathcal{O}_{\mathcal{M}}^{1}}
$$

the Cuntz-Pimsner algebra associated with the $\mathcal{O}_{\mathcal{M}}^{0}$-bimodule $\mathcal{O}_{\mathcal{M}}^{1}$. It is enough to show that $\mathfrak{O}$ is nuclear since $\mathfrak{O}$ is isomorphic the original Cuntz-Pimsner algebra $\mathcal{O}_{\mathcal{M}}$. In fact, $\mathcal{O}_{\mathcal{M}}^{1}$ is a Hilbert 
$\mathcal{O}_{\mathcal{M}}^{0}$-bimodule in $\mathcal{O}_{\mathcal{M}}$ with support $\mathbb{1}$ so that by Proposition 2.3 there exists a monomorphism $I: \mathfrak{O} \hookrightarrow \mathcal{O}_{\mathcal{M}}$. Moreover, since $\mathcal{M} \subset \mathcal{O}_{\mathcal{M}}^{1}$, it follows that $I$ is surjective (cf. [15, Theorem 2.5]).

To show the nuclearity of $\mathfrak{O}$ we need to exploit the additional structure of the bimodule $\mathcal{O}_{\mathcal{M}}^{1}$. Note first that $\mathcal{O}_{\mathcal{M}}^{1}$ also carries a natural left $\mathcal{O}_{\mathcal{M}}^{0}$-valued scalar product given by

$$
\left\langle T^{\prime}, T\right\rangle_{l}:=T^{\prime} T^{*}, \quad T, T^{\prime} \in \mathcal{O}_{\mathcal{M}}^{1}
$$

By a similar argument as before we have that $\mathcal{O}_{\mathcal{M}}^{1}$ is also full w.r.t. $\langle\cdot, \cdot\rangle_{l}$, i.e. $\mathcal{O}_{\mathcal{M}}^{0}=\left\langle\mathcal{O}_{\mathcal{M}}^{1}, \mathcal{O}_{\mathcal{M}}^{1}\right\rangle_{l}$. We conclude that $\mathcal{O}_{\mathcal{M}}^{1}$ can also be interpreted as an $\mathcal{O}_{\mathcal{M}}^{0}$-imprimitivity bimodule (cf. [17, Section 3.1]).

Put

$$
\mathcal{J}:=\mathcal{O}_{\mathcal{M}}^{1} \widehat{\otimes} \mathcal{K} \quad \text { as well as } \quad \mathcal{B}:=\mathcal{O}_{\mathcal{M}}^{0} \otimes \mathcal{K},
$$

where $\widehat{\otimes}$ denotes the external tensor product of Hilbert bimodules and $\mathcal{K}$ is the $C^{*}$-algebra of compact operators over a separable Hilbert space. Then, $\mathcal{J}$ is an imprimitivity $\mathcal{B}$-bimodule (cf. Remark 2.5). Since $\mathcal{B}$ is a stable and separable $C^{*}$-algebra we obtain from Corollary 3.5 in [7] that there is an isomorphism of Hilbert bimodules

$$
\beta_{0}: \mathcal{J} \rightarrow \mathcal{B}
$$

where $\mathcal{B}$ is considered as a bimodule over itself with multiplication as right action and the left action being specified by a suitable automorphism $\theta \in$ Aut $\mathcal{B}$. The isomorphism $\beta_{0}$ extends to an isomorphism of the corresponding Cuntz-Pimsner algebras. Moreover, $\beta_{0}$ also extends to an isomorphism of the associated multiplier algebras. Hence we have

$$
\beta_{0}: \mathcal{O}_{\mathcal{J}} \rightarrow \mathcal{O}_{\mathcal{B}} \simeq \mathcal{B} \rtimes_{\theta} \mathbb{Z},
$$

where for the last isomorphism with the crossed product we use the results in [15, Chapter 1].

Let $E \in \mathcal{K}$ be a minimal projection. Then using Eq. (11) we may define a monomorphism

$$
\beta: \mathcal{O}_{\mathcal{M}}^{1} \rightarrow \mathcal{B} \quad \text { by means of } \quad T \mapsto \beta_{0}(T \otimes E) .
$$

Note that the image of $\mathcal{O}_{\mathcal{M}}^{1}$ generates $\mathcal{B}$ as a $\mathcal{B}$-bimodule and that (by universality) $\beta$ can be extended to a monomorphism

$$
\beta: \mathfrak{O} \rightarrow \mathcal{O}_{\mathcal{B}},
$$

where $\mathfrak{O}:=\mathcal{O}_{\mathcal{O}_{\mathcal{M}}^{1}}$ was introduced in the beginning of the proof. Since $\mathcal{O}_{\mathcal{M}}^{0}$ is nuclear (cf. Proposition 2.6) we have that $\mathcal{B}$ is nuclear and the same is true for the crossed product $\mathcal{B} \rtimes_{\theta} \mathbb{Z}$ ([4, Theorem 15.8.2]). From Eq. (12) we obtain that $\mathcal{O}_{\mathcal{J}}$ and $\mathcal{O}_{\mathcal{B}}$ are nuclear $C^{*}$-algebras.

Finally, we turn our attention to the Cuntz-Pimsner algebra $\mathfrak{O}$. We will conclude the proof by showing that this algebra is a corner of the nuclear algebra $\mathcal{O}_{\mathcal{B}}$ : By Remark 2.5 we may identify $\mathcal{O}_{\mathcal{J}} \simeq \mathfrak{O} \otimes \mathcal{K}$ and using (12) we conclude that $\left\{\beta_{0}(T \otimes V) \mid T \in \mathfrak{O}, V \in \mathcal{K}\right\}$ is total in $\mathcal{O}_{\mathcal{B}}$. Let us now consider the identity $\mathbb{1}$ of the multiplier algebra $M(\mathfrak{O})$. Then, $\mathbb{1} \otimes E \in M(\mathfrak{O} \otimes \mathcal{K})$ and we define the projection $E_{\beta}:=\beta_{0}(\mathbb{1} \otimes E) \in M\left(\mathcal{O}_{\mathcal{B}}\right)$. For $T \in \mathfrak{O}$ we have that $\beta(T)=E_{\beta} \beta(T) E_{\beta}$. On the contrary, take $B=\sum_{i=1}^{n} \beta_{0}\left(T_{i} \otimes V_{i}\right) \in \mathcal{O}_{\mathcal{B}}, T_{i} \in \mathfrak{O}, V_{i} \in \mathcal{K}, i=1, \ldots, n$. Then

$$
E_{\beta} B E_{\beta}=\sum_{i} E_{\beta} \beta_{0}\left(T_{i} \otimes V_{i}\right) E_{\beta}=\sum_{i} \beta_{0}\left(T_{i} \otimes E V_{i} E\right)=\beta_{0}\left(\left(\sum_{i} z_{i} T_{i}\right) \otimes E\right),
$$

where $z_{i} \in \mathbb{C}$ are given by $E V_{i} E=z_{i} E$ (recall that $E$ is minimal). We conclude that $\mathfrak{O}$ is isomorphic to the corner $E_{\beta} \mathcal{O}_{\mathcal{B}} E_{\beta}$. Thus, $\mathfrak{O}$ is nuclear. 


\section{Outlook}

Cuntz-Pimsner algebras provide an important family of examples in the theory operator algebras. Moreover, these algebras, which generalise Cuntz algebras, appear naturally in the extension of Doplicher-Roberts superselection theory (cf. [9]) to the case where the observable algebra has a nontrivial center $\mathcal{Z}$ (see e.g. 1, 2, 3]). In this context the category of canonical endomorphisms is isomorphic to a category whose objects are free $\mathcal{Z}$-bimodules. The Cuntz-Pimsner algebras associated to these $\mathcal{Z}$-bimodules generate a $C^{*}$-algebra $\mathcal{F}$ on which one can realise concretely the dual of a compact group (cf. [14 and references cited therein). The class of algebras considered in the present paper contain the Cuntz-Pimsner algebras that appear in this application. In certain special cases, tensor products of these algebras may also appear in concrete models.

Acknowledgments We are grateful to the DFG-Graduiertenkolleg "Hierarchie und Symmetrie in mathematischen Modellen" for supporting a visit of E.V. to the RWTH-Aachen University. E.V. was also partially supported by the European Network "Quantum Spaces - Noncommutative Geometry" HPRN-CT-2002-00280.

\section{References}

[1] H. Baumgärtel and H. Grundling, Superselection in the presence of constraints, J. Math. Phys. 46 (2005), 082303.

[2] H. Baumgärtel and F. Lledó, Duality of compact groups and Hilbert $C^{*}$-systems, Int. J. Math. 15 (2004), 759-812.

[3] H. Baumgärtel and F. Lledó, Superselection structures for $C^{*}$-algebras with nontrivial center, Rev. Math. Phys. 9 (1997), 785-819.

[4] B. Blackadar, K-theory for operator algebras, Mathematical Sciences Research Institute Publications, Cambridge University Press, Cambridge, 1998.

[5] E. Blanchard, Déformations de $C^{*}$-algébres de Hopf, Bull. Soc. math. France 124 (1996), 141-215.

[6] L.G. Brown, Stable isomorphism of hereditary subalgebras of $C^{*}$-algebras, Pacific J. Math. 71 (1977), 335-348.

[7] L.G. Brown, P. Green, and M.A. Rieffel, Stable isomorphism and strong Morita equivalence of $C^{*}$-algebras, Pacific J. Math. 71 (1977), 349-363.

[8] S. Doplicher, C. Pinzari, and R. Zuccante, The $C^{*}$-algebra of a Hilbert bimodule, Bolletino U.M.I. Serie VIII 1-B (1998), 263-282.

[9] S. Doplicher and J.E. Roberts, Why there is a field algebra with compact gauge group describing the superselection structure in particle physics, Commun. Math. Phys. 131 (1990), 51-107.

[10] S. Doplicher and J.E. Roberts, A new duality for compact groups, Invent. Math. 98 (1989), $157-218$.

[11] E. Germain, Approximation properties for Toeplitz-Pimsner $C^{*}$-algebras, preprint, 2002.

[12] T. Kajiwara, C. Pinzari, and Y. Watatani, Ideal structure and simplicity of the $C^{*}$-algebras generated by Hilbert bimodules, J. Funct. Anal. 159 (1998), 295-322. 
[13] A. Kumjian, On certain Cuntz-Pimsner algebras, Pacific J. Math. 217 (2004), 275-289.

[14] F. Lledó and E. Vasselli, Realization of Hilbert $C^{*}$-systems in terms of Cuntz-Pimsner algebras, in preparation.

[15] M.V. Pimsner, A class of $C^{*}$-algebras generalizing both Cuntz-Krieger algebras and crossed products by Z, Fields Inst. Commun. 12 (1997), 189-212.

[16] C. Pinzari, The ideal structure of Cuntz-Krieger-Pimsner algebras and Cuntz-Krieger algebras over infinite matrices, In Operator Algebras and Quantum Field Theory (Accademia Nationale dei Lincei, Roma, July 1-6, 1996), International Press, Cambridge MA, 1997.

[17] I. Raeburn and D.P. Williams, Morita Equivalence and Continuous-Trace $C^{*}$-algebras, American Mathematical Society, Providence, Rhode Island, 1998. 